\title{
REGION OF CONVERGENCE OF DIRICHLET SERIES WITH COMPLEX EXPONENTS ${ }^{1}$
}

\author{
T. M. GALLIE, JR.
}

Consider the Dirichlet series

$$
\sum_{n=1}^{\infty} c_{n} e^{-\lambda_{n} s}
$$

with complex exponents $\lambda_{n}$. Hille [1] has given a geometrical description, which was somewhat simplified later by Valiron [2], of a convex maximal region outside of which the series does not converge and in the interior of which, in case

$$
\lim _{n \rightarrow \infty}(\log n) / \lambda_{n}=0,
$$

the series converges absolutely. In this paper we give a simpler definition (5) of the maximal region, a generalization (4) of the CauchyHadamard formula to give the distance from an interior point to the frontier of this region, and, as an application of this formula, a generalization (Theorem 2) of Hille's result. This contains a well known relation between the abscissas of simple and absolute convergence for Dirichlet series with positive exponents.

It will be convenient to introduce the notation

$$
d(n, s)=-\left|\lambda_{n}\right|^{-1} \log \left|c_{n} e^{-\lambda_{n} s}\right| .
$$

The geometrical significance of the number $d(n, s)$ is that it is the algebraic distance from the point $s$ of the complex $s$-plane to the line $L_{n}$ on which the absolute value of the term $c_{n} e^{-\lambda_{n}}$ is 1 . Let

$$
d(s)=\liminf _{n \rightarrow \infty} d(n, s)
$$

and

$$
D=\{s: d(s) \geqq 0\} .
$$

It is easy to see that the region $D$ is convex and closed and that the series does not converge outside of $D$. For if $s_{1}$ and $s_{2}$ belong to $D$ and if $s=t s_{1}+(1-t) s_{2}$, where $0 \leqq t \leqq 1$, then

Presented to the Society, December 29, 1955; received by the editors July 6, 1955.

1 This work is a part of the author's doctoral thesis at The Rice Institute. 


$$
d(n, s)=t d\left(n, s_{1}\right)+(1-t) d\left(n, s_{2}\right)
$$

and hence

$$
d(s) \geqq t d\left(s_{1}\right)+(1-t) d\left(s_{2}\right) \geqq 0
$$

and thus $D$ is convex. To see that $D$ is closed, suppose that $s$ is a limit point of points of $D$. Then, given any $\epsilon>0$, there is a point $s_{\epsilon}$ of $D$ such that $\left|s-s_{\epsilon}\right|<\epsilon$ or $d(n, s)>d\left(n, s_{\epsilon}\right)-\epsilon$ or

$$
d(s) \geqq d\left(s_{\epsilon}\right)-\epsilon \geqq-\epsilon,
$$

which shows that $s$ belongs to $D$. Suppose, finally, that $s$ does not belong to $D$ or, in other words that $d(s)<0$. Then there exists an $\epsilon>0$ and a sequence of positive integers $n_{i}$ such that $d\left(n_{i}, s\right)<-\epsilon$ for $i=1,2 \cdots$. Since

$$
\left|c_{n_{i}} e^{-\lambda_{n i} s}\right|=e^{-\left|\lambda_{n i}\right| d\left(n_{i}, s\right)}>e^{\left|\lambda_{n i}\right| \epsilon}>1,
$$

we see that the series does not converge at the point $s$.

Slightly less obvious is the proof of the following:

TheOREM 1. If $s$ belongs to $D$, then the distance from $s$ to the frontier of $D$ is $d(s)$.

Let us denote the distance in question by $K$ and $d(s)$ by $R$. We shall show first that $K \geqq R$. Since this is trivially true for $R=0$, assume $R>0$. Given any $\epsilon>0$, we have $d(n, s)>R-\epsilon$ and hence $d\left(n, s^{\prime}\right)>\epsilon$ for $n$ sufficiently large and $\left|s-s^{\prime}\right|<R-2 \epsilon$, which shows that $s^{\prime}$ belongs to $D$ if $\left|s-s^{\prime}\right|<R$, and hence that $K \geqq R$.

On the other hand, if we denote the foot of the perpendicular from $s$ on the line $L_{n}$ by $s_{n}$, since there exists a sequence of positive integers $n_{i}$ such that $\lim _{i \rightarrow \infty} d\left(n_{i}, s\right)=R$, we have

$$
\lim _{i \rightarrow \infty}\left|s-s_{n_{i}}\right|=R \text {. }
$$

Now the sequence of points $s_{n_{i}}$ has at least one limit point $s_{0}$ and it is impossible that $\left|s-s_{0}\right|>R$. Clearly, also, $d\left(s_{0}\right) \leqq 0$.

Suppose $s_{0}$ is an interior point of $D$. Then there exists an equilateral triangle with center at $s_{0}$ and vertices $v_{1}, v_{2}$, and $v_{3}$ which belong to $D$. Denote the length of the medians by $m$. If $n$ is sufficiently large, then $d\left(n, v_{i}\right)>-m / 4$ for $i=1,2,3$, and hence $d\left(n, s_{0}\right)>m / 12$, or $d\left(s_{0}\right)>0$. Since this is a contradiction, $s_{0}$ can not be an interior point. But, from the first paragraph of this proof and the fact that $D$ is closed, it follows that $s_{0}$ belongs to $D$. Thus $s_{0}$ is a frontier point of $D$ and hence $K \leqq R$, which completes the proof.

An application of this theorem is the following. 
THEOREM 2. The series converges absolutely to an analytic function at every point of $D$ whose distance from the frontier of $D$ is greater than

$$
L=\limsup _{n \rightarrow \infty}(\log n) /\left|\lambda_{n}\right| \text {. }
$$

Suppose the distance from a point $s$ of $D$ to the frontier of $D$ is greater than $L$. Then there exists a number $\epsilon>0$ sufficiently small that $d(n, s)>L+3 \epsilon$ for $n$ sufficiently large and hence $d\left(n, s^{\prime}\right)>L+2 \epsilon$ if $\left|s-s^{\prime}\right| \leqq \epsilon$. Since $\left|\lambda_{n}\right|>(L+\epsilon)^{-1} \log n$ for $n$ sufficiently large,

$$
\left|c_{n} e^{-\lambda_{n} s^{\prime}}\right|=e^{-\left|\lambda_{n}\right| d\left(n, s^{\prime}\right)}<n^{-(L+2 \epsilon) /(L+\epsilon)}
$$

from which we conclude that the series converges absolutely and uniformly on the disc $\left|s-s^{\prime}\right| \leqq \epsilon$.

\section{BIBLIOGRAPHY}

1. E. Hille, Note on Dirichlet's series with complex exponents, Ann. of Math. vol. 25 (1924) pp. 261-278.

2. G. Valiron, Sur les solutions des équations différentielles linéaires d'ordre infini et a coefficients constants, Ann. Ecole Norm. (3) vol. 46 (1929) pp. 25-53.

THE RICE INSTITUTE AND

DUKE UNIVERSITY 